\title{
- The Desideratum of Nanomaterials for DNA Sensing
}

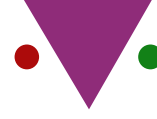

IJCRR

Section: Healthcare

ISI Impact Factor

(2019-20): 1.628

IC Value (2019): 90.81

SJIF (2020) $=7.893$
Anurag Roy

Environmental and Sustainability Institute, University of Exeter, Penryn Campus, Cornwall TR1o gFE, United Kingdom.

E-mail: A.Roy3o@exeter.ac.uk
Along with the evolution of the nanoworld landscape, new factors have emanated to tailor nanomaterials' properties to maximize their potential to secure the overall goal of integrating nanomaterials into critical technologies that directly or indirectly reinforce to yield societal benefit. The bio-medical area has also taken its place in this field on this journey from nanomaterial synthesis and characterization to application. The classic combination of nanomaterials and the most desired component for next-generation technologies such as deoxyribonucleic acid (DNA) moves from the sphere of dreams to the panorama of modern science and technology. It opens the door to novel bio and nanotechnology applications. Apart from the technological aspect of nano-biotechnology, a critical understanding of how nanomaterials interact to DNA of different compositions and how it impacts DNA sensing has recently aroused the research community's attention. Following the developments in this field, it has become apparent that nanomaterials' exploration and diversity in DNA sensing are closely associated with nanomaterials' physicochemical properties and structural aspects of DNA. Several novel biotechnologies based on fundamental aspects of nanomaterial-DNA binding and their interactions have been developed in various fields such as in the design of new types of sensing material, nanodevices as well as in diagnostic application, gene therapy, drug delivery application, food industry, bio-electronics, bio-computation and forensic sciences in recent years. In addition to the knowledge of chemical and structural properties (biocompatibility, water solubility and biodegradability) of nanomaterials, more efforts have been directed towards identifying and designing nanomaterials for site-specific and sequence-specific DNA binding. For medical technology, high sensitivity of the nanomaterial-DNA binding is also essential. Only one-to-one interactions between a single DNA molecule and nanoparticles can achieve high sensitivity through a specific or nonspecific molecular binding. A significant part of the nanomaterial-DNA interaction study deals with the functionalized gold, silver nanoparticles (NPs) conjugation with thiolated oligonucleotides for specific DNA hybridization and identification of complementary sequences of interest.

Interestingly, the non-covalent binding between nanomaterials and DNA is required to directly release nucleic acids in gene therapy and regulation. More importantly, genetic functions of DNA, such as transcription, replicationand repair processes, even the entire genome processes, can be affected by nanomaterial-DNA binding. Expressly, NPs that bind to DNA with a high affinity could prohibit the normal functions of some critical DNA-binding proteins, such as RNA polymerase and DNA polymerase, by occupying protein-binding sites and impeding the movement of protein along with the DNA, which could result in competitive inhibition of genetic functions. Besides, nanoparticle binding to DNA also raises concerns about DNA-associated enzymes and metabolism in a biological cell as a whole system. However, in some cases, DNA structure deformity and aggregation induced by carbon NP binding that affects DNA bioactivity pose challenges for developing DNA-nanoparticle-based technologies for medicinal and therapeutic applications. Therefore, further intensive research work needs to focus on nanomaterials' interaction other than gold, silver, and carbon with DNA. The importance and fundamental aspects of nanomaterials in DNA sensing are an excellent and broader interdisciplinary research scope. Also, nanomaterials employment for DNA sensing opens a new perspective for developing DNA in technological. 